\title{
Dynamics of Field Line Mappings in Magnetic Flux Tubes
}

\author{
Grzegorz Graff' ${ }^{1}$ [ . Justyna Signerska-Rynkowska' ${ }^{1}$ (1)
}

Received: 19 December 2017 / Accepted: 13 August 2018 / Published online: 25 August 2018

(C) The Author(s) 2018

\begin{abstract}
We study the topological constraints on the dynamics of magnetic field lines in flux tubes. Our approach is based on the application of the topological invariant: fixed point index. We consider periodic flux tubes and find various restrictions on the field lines that come from the sequence of fixed point indices of iterations. We also analyze the case of a tube with a cylindrical obstacle, deducing some special dynamical properties of the field line mapping from geometrical properties of the tube and the additional assumptions put on the field.
\end{abstract}

Keywords Magnetic flux tubes - Fixed point index · Diffeomorphisms · Low-dimensional dynamics

Mathematics Subject Classification (2010) 37C25 55 M20 $\cdot 37$ C05 $\cdot 76$ W05

\section{Introduction}

Magnetic flux tubes are one of the most important structures used in description and analysis of the evolution of astrophysical magnetic fields. The interest in study of fixed point index for field line mappings in magnetic flux tubes comes from induced topological constraints on the dynamics of the field. Namely, according to the theory of

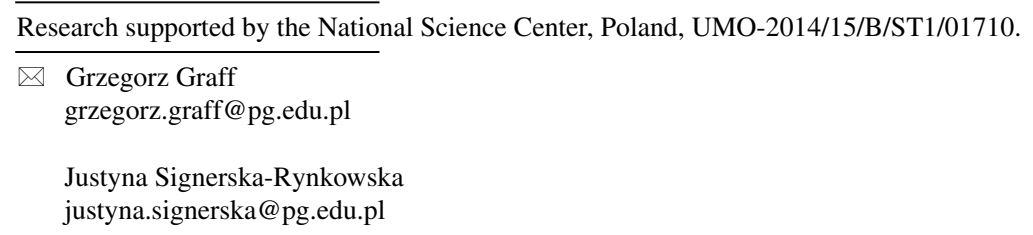

1 Faculty of Applied Physics and Mathematics, Gdańsk University of Technology, ul. Narutowicza 11/12, 80-233 Gdańsk, Poland 
Taylor [20] concerning magnetohydrodynamics of toroidal plasmas, the topological constraints are gradually relaxed until so-called linear force-free final state.

However, recent works (see e.g. [18, 23, 24] and references therein) show that this does not necessarily apply to the magnetohydrodynamics of the solar corona. This observation has been explained by the additional topological constraints coming from fixed point index theory, which prevent from reaching the final state predicted by Taylor's theory.

In case of flux tubes instead of considering the continuous flow of the field through the tube, one can analyze the discrete field line mapping through the consecutive horizontal cross-sections of the tube: $f: D \rightarrow D$, where $D$ is a two dimensional closed disk. Consequently, the methods of discrete dynamical systems apply, which enable one to examine a topological invariant for $f$ called fixed point index.

Let us mention here that fixed point index could be successfully applied for topological characterization of physical objects called vortices that are used in description of many real physical systems (Bose-Einstein condensates, superfluid helium, coherent optical fields, singular electron microscopy and others) [5, 8, 21].

Assuming that the flow lines preserve the boundary of a general flux tube, the sum of indices of interior fixed points is invariant under the evolution of the field. Analogously, one can consider periodic point indices for a periodic flux tube (fixed point indices of iterated mappings), which, although potentially lead to infinitely many such invariants, give rise to at most two types of independent dynamical constraints (see Theorem 4.2 in [23]).

In the current paper we generalize mathematical approach from [23] in two directions. First, we analyze dynamics of field line mappings under weaker assumptions put on the field itself, obtaining the same types of results. Namely, basing on recent theory developed in [11] and the smooth index theory (cf. [2, 4]) we obtain results similar to those of Yeates and Hornig in [23] without demanding non-degeneracy of fixed/periodic points (hard to verify in practice), and also dropping the assumption on the vanishing of the field divergence (Theorems 3 and 4).

Second, we also study vector fields not only in cylindrical tubes but also in annular ones, using the geometry of the obstacle inside the tube to obtain additional information about the dynamics of the field. This approach gives us some types of bounds which come from fixed point indices (Theorems 5, 6 and 7), but also other types of dynamical restrictions. Basing on the results of Franks (related to the rotation theory for an area preserving annulus homeomorphism [6], our Theorem 8), we analyze special properties of the magnetic field mapping in the annular tube. In particular, we formulate the assumption on the vector field imposing that the induced annular map is an area preserving diffeomorphism, in which case the existence of one periodic point implies the existence of infinitely many periodic points (Proposition 1). Therefore rotation theory also proves to be useful in studying of systems arising in magnetohydrodynamics, as well as in other natural sciences (see e.g. [19] for neuroscience applications). In our analysis the effective tool for detecting the existence of periodic points is the so-called rotation number of a point. This part of our findings is illustrated with suitable examples in the final section. 


\section{Cylindrical Flux Tube with no Obstacles}

\subsection{Assumptions on the Field}

Firstly we state all the necessary physical assumptions on the magnetic field and their counterparts for the non-obstacle case.

After [23] the magnetic field will be denoted

$$
\mathbf{B}(\mathbf{x})=B^{r} \mathbf{e}_{\mathbf{r}}+B^{\varphi} \mathbf{e}_{\varphi}+B^{z} \mathbf{e}_{\mathbf{z}}
$$

where $\mathbf{x}=(r, \varphi, z)$ are the coordinates of a point in the cylinder $\mathcal{C}=\{(r, \varphi, z): 0 \leqslant$ $r \leqslant R, 0 \leqslant \varphi \leqslant 2 \pi, 0 \leqslant z \leqslant 1\}$ with radius $R$ and height 1 . The lower and upper boundary discs of the cylinder (corresponding to $z=0$ and $z=1$, respectively) will be denoted by $D_{0}$ and $D_{1}$. The boundary conditions of the field $\mathbf{B}$ are the following:

$$
\begin{aligned}
& \mathbf{B} \neq 0, \\
& \left.B^{r}\right|_{r=R}=0, \\
& B^{z}(r, \varphi, z)>0, \quad \text { for all }(r, \varphi, z) \in \mathcal{C}, \\
& \left.\mathbf{B}\right|_{D_{0}}=\left.\mathbf{B}\right|_{D_{1}} .
\end{aligned}
$$

We also assume that $\mathbf{B}$ is $C^{1}$. The (1a) means that the magnetic field never completely vanishes. The second assumption (1b) means that the lateral surface (side boundary) of the cylinder is invariant for the trajectories of the field (no crossing of the surface). Equation (1c) guarantees that the field always points up and that its lines can be parametrized with $z$ ( $z$ acting as the time-variable), while (1d) is the periodicity condition (the distribution of the normal magnetic field is the same across both ends of the tube) which allows later for iterations of field line mappings. Such periodicity occurs e.g. in flux tubes with toroidal geometry - see [23] and references therein. Moreover, we expect that the theory developed in this paper can also be used in analysis of solar coronal magnetic loops since numerical simulations of these loops sometimes are periodic and in general it is possible to turn the aperiodic flux tube into a periodic domain using so-called reference field (similar to the relative magnetic helicity defined in [3]).

We remark that instead of the two our conditions (1c)-(1d), in [23] we have only the requirement that $\left.B^{z}\right|_{D_{0}}=\left.B^{z}\right|_{D_{1}}>0$. However, if the variable $z$ is going to be interpreted as "time", then it is clear that $B^{z}$ must be positive everywhere in $\mathcal{C}$. Similarly the "periodicity condition" on $D_{0}$ and $D_{1}$, which causes that taking iterations of the field line mapping $f$ (to be defined below) makes sense, also needs to hold not only with respect to $B^{z}$ but to the whole $\mathbf{B}$. Moreover, the assumptions (1c)-(1d) are satisfied by all the examples studied in [23], as well as they are implicitly present in some proofs therein. Therefore considering (1a)-(1d) does not reduce generality of our considerations.

Considering iterations we need to reformulate the assumption (1d) in the following way:

$$
\mathbf{B}(r, \varphi, z)=\mathbf{B}(r, \varphi, z+1) \text { for all }(r, \varphi, z) \text { such that } z \in[0, \infty) .
$$


Note that (1d') is only an extension of the toroidal dynamics (i.e. the dynamics within the bounded tube, $0 \leqslant z \leqslant 1$ ) onto the periodic unbounded tube due to the periodicity of the vector field.

Another important assumption is the vanishing divergence of the field:

$$
\operatorname{div} \mathbf{B}(r, \varphi, z):=\nabla \cdot \mathbf{B}=0,
$$

where

$$
\nabla \cdot \mathbf{B}=\frac{1}{r} \frac{\partial}{\partial r}\left(r B^{r}(r, \varphi, z)\right)+\frac{1}{r} \frac{\partial B^{\varphi}}{\partial \varphi}(r, \varphi, z)+\frac{\partial B^{z}}{\partial z}(r, \varphi, z) .
$$

Equation (2) means that the magnetic flux is the same through any horizontal crosssection of the cylinder. In the context of real magnetic fields condition (2) must always be satisfied because otherwise such a field would violate Maxwell's equations and would be unphysical. However, note that for our developments we do not assume (2) in general, unless stated otherwise.

In our setting the variable $z$ can be interpreted as "time". Indeed, considering the original autonomous system

$$
\left\{\begin{array}{l}
\dot{r}=B^{r}(r, \varphi, z), \\
\dot{\varphi}=B^{\varphi}(r, \varphi, z), \\
\dot{z}=B^{z}(r, \varphi, z),
\end{array}\right.
$$

and dividing the coordinates of the field $\mathbf{B}$ by $B^{z}$ (making use of the fact that $B^{z}>0$ ) we obtain two-dimensional system

$$
\left\{\begin{array}{l}
\frac{d r}{d z}=\hat{B}^{r}(r, \varphi, z) \\
\frac{d \varphi}{d z}=\hat{B}^{\varphi}(r, \varphi, z)
\end{array}\right.
$$

where $\hat{\mathbf{B}}=\left(\hat{B}^{r}, \hat{B}^{\varphi}, \hat{B}^{z}\right)$ with $\hat{\mathbf{B}}:=\frac{\mathbf{B}}{B^{z}}$. This system is in general not autonomous, as we allow $B^{r}, B^{\varphi}$ and $B^{z}$ to depend on $z$ explicitly. However, it can be viewed as the following three-dimensional autonomous system:

$$
\left\{\begin{array}{l}
\dot{r}=\hat{B}^{r}(r, \varphi, z) \\
\dot{\varphi}=\hat{B}^{\varphi}(r, \varphi, z) \\
\dot{z}=1
\end{array}\right.
$$

which is equivalent to the original system.

Now we define field line mappings (for $\hat{\mathbf{B}}$ ) $f: x_{0} \mapsto f\left(x_{0}\right)$, assigning to a point $x_{0}=\left(r_{0}, \varphi_{0}\right)$ an endpoint of the magnetic line starting at $\left(x_{0}, 0\right) \in D_{0}$ and ending at $\left(f\left(x_{0}\right), 1\right) \in D_{1}$. Since all magnetic field lines (being integral curves of $\left.\mathbf{B}\right)$ connect $D_{0}$ with $D_{1}$ in the same direction and are parametrized with $z, 0 \leqslant z \leqslant 1$, we obtain that

$$
F\left(x_{0}, \hat{z}\right):=\int_{0}^{\hat{z}} \frac{\mathbf{B}\left(F\left(x_{0}, z\right)\right)}{B^{z}\left(F\left(x_{0}, z\right)\right)} d z, \hat{z} \in[0,1]
$$

is the point of intersection of the field line starting at $x_{0} \in D_{0}$ for $z_{0}=0$ with the section $\{z=\hat{z}\}$ (where $x_{0}=\left(r_{0}, \varphi_{0}\right)$ and $\left.F\left(x_{0}, \hat{z}\right)=\left(r_{\hat{z}}, \varphi_{\hat{z}}\right)\right)$. This gives the definition

$$
f\left(x_{0}\right):=F\left(x_{0}, 1\right) .
$$

Note that in the view of (4), $f$ can be identified with the time-1 map $\Phi(1, \cdot)$ induced by the flow $\Phi(\cdot, \cdot)$ arising from (4) for an initial condition $x_{0}$ on the lower 
disc $D_{0}$. Further, notice that $f: D_{0} \rightarrow D_{1}$ is an orientation preserving diffeomorphism of the disc $D=D_{0}=D_{1}$ to itself (we assume that $B$ is differentiable and time-1 maps of smooth vector fields of bounded domains of $\mathbb{R}^{n}$ are orientation preserving diffeomorphisms, see e.g. [1])

\subsection{Topological Constraints on the Evolution of the Magnetic Field Given by Fixed Point Indices of Iterations}

Fixed point index is a well-known topological invariant that characterizes an isolated fixed point. The sequence of fixed point indices of iterates is a powerful tool that was used in various contexts in periodic point theory and dynamics, cf. for example [2, 4, 9-11].

Let $f: \mathbb{R}^{2} \rightarrow \mathbb{R}^{2}$ and $p$ be an isolated fixed point of $f$. Then the fixed point index, ind $(f, p)$, is defined in the following way: we take a small neighborhood $W$ of $p$ without any other fixed points (except for $p$ ) and take a circle $C \subset W$ centered at $p$. Then ind $(f, p)$ is equal to the number of revolutions of the vector connecting $z$ with $f(z)$ as the point $z$ is running one revolution along $C$ in the positive direction.

We can define ind $(f, p)$ also in case $f: D \rightarrow \mathbb{R}^{2}$ and $p \in \partial D$. Let $W \subset \mathbb{R}^{2}$ be some neighborhood of $p$. Then $\operatorname{ind}(f, p):=\operatorname{ind}(\tilde{f}, p)$, where $\tilde{f}=f \circ r$ and $r$ is a retraction of $W$ onto $D$ (cf. [16]). For example, assuming $(0,0)$ is the center of $D$ of radius $R$ we can take $W=\mathbb{R}^{2}$ and $r(x)=R \frac{x}{|x|}$ and then:

$$
\tilde{f}(x)= \begin{cases}f(x) & \text { if }|x| \leqslant R \\ f\left(R \frac{x}{|x|}\right) & \text { if }|x|>R\end{cases}
$$

Note that the map $\tilde{f}$ might be not differentiable even if $f$ has this property. In our considerations by a $C^{1}$ map we understand a map which has continuous partial derivatives also at the points belonging to the boundary of $D$ (see Definition 1).

By Fix $(f)$ we denote the set of fixed points of $f$. By $T_{\text {int }}^{1}$ and $T_{\partial}^{1}$ we denote, respectively, the sum of the indices of fixed points in the interior of the disc $D$ and on the boundary $\partial D=S^{1}$ :

$$
T_{\mathrm{int}}^{1}=\sum_{p \in \operatorname{Fix}(f) \cap \operatorname{Int}(D)} \operatorname{ind}(f, p) ; \quad T_{\partial}^{1}=\sum_{p \in \operatorname{Fix}(f) \cap \partial D} \operatorname{ind}(f, p) ; \quad T^{1}:=T_{\mathrm{int}}^{1}+T_{\partial}^{1} .
$$

We recall that $T^{1}$ is a homotopy invariant. In particular, if $f$ is a continuous mapping of the disc, then $T^{1}=1$ (as $D$ is contractible and therefore any continuous map is homotopic to the constant map $g \equiv 0$ which has a single fixed point at $x_{0}=0$ with index +1$)$.

Similarly, if $x_{0}$ is a periodic point of $f$ with period $q$, then it is the fixed point of $f^{q}$ and therefore one can define its fixed point index $\operatorname{ind}\left(f^{q}, x_{0}\right)$. Similarly as for $q=1$, we define $T_{\text {int }}^{q}, T_{\partial}^{q}$ and get:

$$
T^{q}:=T_{\text {int }}^{q}+T_{\partial}^{q}=1
$$

We remark that an alternative way to obtain (7) would be to use Poincaré-Hopf theorem (compare with the proof of Lemma 4). 
Now, we will prove Lemma 1, which has strong implications in the description of properties of the field line map. This result was obtained in [23] under the condition that all periodic points are non-degenerate as well as under the assumption (2) stating that the divergence is zero. For a fixed point $x_{0}$ (which is in the interior of $D$ ) the condition on non-degeneracy means that $\operatorname{det}\left(\mathrm{D} f^{q}\left(x_{0}\right)-\mathrm{Id}\right) \neq 0$ for each natural $q$. However in our generalization below we drop both of these assumptions.

For the rest of this section we make another general assumption: the set $\operatorname{Fix}\left(f^{q}\right)$ (where $f: D \rightarrow D$ is a field line mapping) is finite for every $q \in \mathbb{N}$. Note that this is equivalent to the fact that every periodic point of $f$ is isolated (as $D$ is a compact set).

Lemma 1 (cf. Lemma 3.3 [23]) Let $f$ be the field line mapping of the magnetic field $\mathbf{B}$ in the cylindrical flux tube, with the conditions (1a)-(1d') and finite set Fix $\left(f^{q}\right)$ for every $q \in \mathbb{N}$. Then for every fixed point $x_{0} \in \partial D$ we have

$$
\operatorname{ind}\left(f^{q}, x_{0}\right)=\operatorname{ind}\left(f, x_{0}\right) \text { for all } q \in \mathbb{N} .
$$

Before giving the proof of Lemma 1 we state Lemma 2 below. We use the following notations:

$$
\begin{aligned}
& \mathbb{R}_{+}^{m+1}=\left\{(x, t) \in \mathbb{R}^{m} \times \mathbb{R}: t \geq 0\right\}, \\
& \mathbb{R}_{0}^{m+1}=\left\{(x, t) \in \mathbb{R}^{m} \times \mathbb{R}: t=0\right\} .
\end{aligned}
$$

Definition 1 By a $C^{1}$ map we understand a map which has continuous partial derivatives of degree 1 , where for a point $x_{0} \in \mathbb{R}_{0}^{m+1}$ we consider right derivatives $\frac{\partial_{+} f_{i}\left(x_{0}\right)}{\partial t}$, $i=1, \ldots, m+1$.

We denote by $D:=D f(0,0)$ the derivative of $f$ at $(0,0) \in \mathbb{R}^{m} \times \mathbb{R}_{+}$.

Lemma 2 (Lemma 3.3 in [11]) Let $f: \mathbb{R}_{+}^{m+1} \rightarrow \mathbb{R}_{+}^{m+1}$ be a $C^{1}$ map such that $f\left(\mathbb{R}_{0}^{m+1}\right) \subset \mathbb{R}_{0}^{m+1}$ and let $(0,0) \in \mathbb{R}^{m+1}$ be an isolated fixed point of $f$ such that $\sum_{j=0}^{q-1} D^{j}$ is nonsingular. Then $(0,0)$ is an isolated fixed point of $f^{q}$ and

$$
\operatorname{ind}\left(f^{q},(0,0)\right)=\rho \cdot \operatorname{ind}(f,(0,0)),
$$

where $\rho=\operatorname{sign} \operatorname{det}\left(\sum_{j=0}^{q-1} D^{j}\right)$.

Proof of Lemma 1 In our case $m=1$ and, as the boundary of the upper half-plane $\mathbb{R} \times \mathbb{R}_{+}$is locally homeomorphic to $\partial D$, we will identify $x_{0}$ with $(0,0)$, Int $D$ with $\operatorname{Int}\left(\mathbb{R} \times \mathbb{R}_{+}\right)$and $\partial D$ with $\partial\left(\mathbb{R} \times \mathbb{R}_{+}\right)$. We represent our map $f$, defined in (5), locally near the fixed point $x_{0} \in \partial D$ as $f(x, t)=\left(f_{1}(x, t), f_{2}(x, t)\right)$, where $x \in \mathbb{R}$ and $t \geqslant 0$.

First observe that, as $f$ preserves the boundary, the derivative of $f$ at $(0,0)$ has the form

$$
D=\left[\begin{array}{ll}
a & \cdot \\
0 & b
\end{array}\right],
$$


where $a=\frac{\partial f_{1}}{\partial x}(0,0)$ and $b=\frac{\partial f_{2}}{\partial t}(0,0)$. Notice that $b \geqslant 0$ by the definition (we are in $\mathbb{R} \times \mathbb{R}_{+}$). Furthermore, $f$ is a diffeomorphism that preserves the orientation so we obtain that $a>0$ and in fact $b>0$.

Thus we obtain that for each $q$

$$
\operatorname{det} \sum_{j=0}^{q-1} D^{j}=\sum_{j=0}^{q-1} a^{j} \cdot \sum_{j=0}^{q-1} b^{j}>0 .
$$

As a consequence, by Lemma 2, we get that for a fixed point $(0,0)$ :

$$
\operatorname{ind}\left(f^{q},(0,0)\right)=\operatorname{ind}(f,(0,0)) .
$$

This completes the proof of Lemma 1.

Definition 2 For a given $d \in \mathbb{N}$ we define the basic periodic sequence:

$$
\operatorname{reg}_{d}(q)=\left\{\begin{array}{lll}
d & \text { if } & d \mid q, \\
0 & \text { if } & d \backslash \chi q
\end{array}\right.
$$

The representation of a given sequence as the sum of basic periodic sequences is called a periodic expansion.

Using the language of periodic sequences we may express the equality (12) as:

$$
\operatorname{ind}\left(f^{q},(0,0)\right)=a_{1} \operatorname{reg}_{1}(q),
$$

where $a_{1}=\operatorname{ind}(f,(0,0))$.

In contrast to simple behavior of indices for the boundary fixed points, the behavior of indices of an interior fixed point can be more complicated. The following theorem was proved in [2] (Theorem 3.7) and gives the possible periodic expansions for fixed point indices at fixed points. Let us denote by $\sigma(D f(p))$ the spectrum of derivative of $f$ at $p$.

Theorem 1 Let $f$ be a planar orientation preserving diffeomorphism.

(1) There can be only two forms of local indices of iterations at the fixed point $p \in \operatorname{Fix}(f)$ :

$(\alpha) \quad \operatorname{ind}\left(f^{q}, p\right)=a_{1} \operatorname{reg}_{1}(q)$, if there are no roots of unity of degree greater than 1 in $\sigma(D f(p))$

$(\beta) \quad \operatorname{ind}\left(f^{q}, p\right)=\operatorname{reg}_{1}(q)+a_{d} r e g_{d}(q)$, if there is a primitive root of unity of degree $d>1$ in $\sigma(D f(p))$,

where $a_{i} \in \mathbb{Z}(i=1, d), d \geqslant 2$ in all cases.

(2) Every sequence of one of the forms $(\alpha)$ or $(\beta)$ can be realized as $\left\{\operatorname{ind}\left(f^{q}, p\right)\right\}_{q=1}^{\infty}$ for some local diffeomorphism $f$ of $\mathbb{R}^{2}$.

For $k \geqslant 1$ we define $P^{k}(f)=\operatorname{Fix}\left(f^{k}\right)$ and $P_{k}(f)=P^{k}(f) \backslash \bigcup_{0<n<k} P^{n}(f)$. The former is the set of $k$-periodic points of $f$. If $P_{k}(f) \neq \emptyset$ then $k$ is called a minimal period of $f$. The set of all minimal periods of $f$ will be denoted by $\operatorname{Per}(f)$ and the set of all periodic points of $f$ by $\mathrm{P}(f)$. 
Let $p \in P_{k}$, then the $k$-orbit of $p$ is defined as the set $O_{p}=$ $\left\{p, f(p), \ldots, f^{k-1}(p)\right\}$. The sequence of indices for an orbit (equal to the sum of indices of periodic points in the orbit) can be easily deduced from Theorem 1 by replacing each $\mathrm{reg}_{d}$ (including $\mathrm{reg}_{1}$ ) by $\mathrm{reg}_{k d}$ (cf. Lemma 2.10 in [12]). Namely:

Theorem 2 Let $f$ be a planar orientation preserving diffeomorphism.

(1) There can be only two forms of local indices of iterations at $k$-periodic orbit $O_{p}$ :

$\left(\alpha^{\prime}\right)$ ind $\left(f^{q}, O_{p}\right)=a_{k} r e g_{k}(q)$, if there are no roots of unity of degree greater than 1 in $\sigma\left(D f^{k}(p)\right)$.

$\left(\beta^{\prime}\right) \quad$ ind $\left(f^{q}, O_{p}\right)=\operatorname{reg}_{k}(q)+a_{k d} r e g_{k d}(q)$, if there is a primitive root of unity of degree $d>1$ in $\sigma\left(D f^{k}(p)\right)$,

where $a_{i} \in \mathbb{Z}(i=k, k d), d \geqslant 2$ in all cases.

(2) Every sequence of one of the forms $\left(\alpha^{\prime}\right)$ or $\left(\beta^{\prime}\right)$ can be realized as $\left\{\operatorname{ind}\left(f^{q}, O_{p}\right)\right\}_{q=1}^{\infty}$ for some local diffeomorphism $f$ of $\mathbb{R}^{2}$, where $p$ is a periodic point of $f$.

In the further part of the paper we will use the following well-known fact (cf. for example [14]).

Lemma 3 The set of minimal periods of an orientation preserving circle homeomorphism consists of one element or is the empty set.

Observe now that in our context, by Lemma $3, \operatorname{Per}\left(f_{\mid \partial D}\right)=\emptyset$ or $\operatorname{Per}\left(f_{\mid \partial D}\right)=\{k\}$ $\left(k=1,2, \ldots\right.$ is a given fixed period). In the last case denote by $x_{i}$ periodic points of $f$ in $\partial D$. Then the sequence of indices of $\left(f^{k}\right)^{n}$ at each $x_{i}$ is constant by Lemma 1 , i.e.

$$
\operatorname{ind}\left(\left(f^{k}\right)^{n}, x_{i}\right)=a_{k}^{i},
$$

where $a_{k}^{i}=\operatorname{ind}\left(f^{k}, x_{i}\right)$ (and $k$ is fixed). Of course, if $k \nmid q$ then $\operatorname{ind}\left(f^{q}, x_{i}\right)=0$. Finally, summing over all points $x_{i}$ we get that

$$
T_{\partial}^{q}=\sum_{i} a_{k}^{i} \operatorname{reg}_{k}(q) .
$$

We arrive at the important result:

Theorem 3 (cf. Theorem 4.2 in [23]) If $f$ is the field line map of the magnetic field over cylindrical flux tube with our assumptions (1a)-(1d'), then

a) if $T_{\text {int }}^{1} \neq 1$, then $T_{\text {int }}^{q}=T_{\text {int }}^{1} \neq 1$ for all $q \in \mathbb{N}$,

b) if $T_{\text {int }}^{1}=1$, then $T_{\text {int }}^{q}=1$ for all $q \in \mathbb{N}$ iff $\sum_{i} a_{k}^{i}=0$.

Proof Case a). If $T_{\text {int }}^{1} \neq 1$, then $T_{\partial}^{1} \neq 0$, thus there exists a fixed point $p$ of $f$ in $\partial D$, which means by Lemma 3 that the set of minimal periods $\operatorname{Per}(f)=\{1\}$, i.e the set of periodic points of $f$ consists only of fixed points. Consequently,

$$
T_{\partial}^{q}=\sum_{p \in \operatorname{Fix}(f) \cap \partial D} \operatorname{ind}\left(f^{q}, p\right)=\sum_{p \in \operatorname{Fix}(f) \cap \partial D} \operatorname{ind}(f, p)=T_{\partial}^{1},
$$


where in the second equality we used Lemma 2.

By the formulas (7) and (16) we obtain:

$$
T_{\mathrm{int}}^{q}=1-T_{\partial}^{q}=1-T_{\partial}^{1}=T_{\mathrm{int}}^{1},
$$

which ends the proof of the Case a).

Case b). Again, we have $T_{\text {int }}^{q}=1-T_{\partial}^{q}$. Thus $T_{\text {int }}^{q}=1$ is equivalent to $T_{\partial}^{q}=0$ i.e. to $\sum_{i} a_{k}^{i}=0$.

Remark 1 If there are no periodic points on the boundary, then the condition $\sum_{i} a_{k}^{i}=$ 0 of item b) of Theorem 3 is automatically satisfied. To verify this condition one may use rotation number. Let us recall that irrationality of the rotation number of an orientation preserving circle homeomorphism is equivalent to the absence of periodic points ([14]). The rotation number is easily computed as

$$
\varrho(f):=\lim _{n \rightarrow \infty} \frac{\tilde{f}^{n}(x)-x}{n} \bmod 1,
$$

where $x \in \mathbb{R}$ is arbitrary initial condition and $\tilde{f}$ denotes any lift of the map $f$ to $\mathbb{R}$ with respect to the projection $\mathfrak{p}: t \mapsto \mathrm{e}^{2 \pi \imath t} \in S^{1}$.

If the condition $\sum_{i} a_{k}^{i}=0$ in the case b) of Theorem 3 is not satisfied, there are additional constraints for the magnetic field which follow from iterations. Now we define a quantity that is an invariant for a higher order iterations and gives some constraints on the field evolution.

First we define the notion of $\gamma_{n}$-periodic points (for the related definition of socalled $\delta_{m}$-periodic points see $\left.[13,17]\right)$.

Definition 3 A periodic point $x$ of $f$ with minimal period $k$ is said to be a $\gamma_{n}$-periodic point $(n>1)$ if $n=k d$, where $d$ denotes the degree of a primitive root of unity in $\sigma\left(D f^{k}(x)\right)$.

Theorem 4 If $f$ is the field line map of the magnetic field over cylindrical flux tube and $n>1$ is the minimal period for boundary periodic points of $f$ with non-zero indices, then $f$ has either an n-periodic point or $\gamma_{n}$-periodic point in IntD.

Proof We can rewrite the equation $1-T_{\partial}^{q}=T_{\text {int }}^{q}$ in the form:

$$
\operatorname{reg}_{1}(q)-A_{n} \operatorname{reg}_{n}(q)=\sum_{p \in \operatorname{Fix}(f) \cap \operatorname{Int} D} \operatorname{ind}\left(f^{q}, p\right)
$$

where in the left hand-side there are terms related to the periodic expansions of the orbits in $\partial D$ (notice that by the formula (15) $A_{n}=\frac{1}{n} \sum_{i} a_{n}^{i}, A_{n} \neq 0$ ).

The term $\operatorname{reg}_{n}(q)$ must be realized by a periodic expansion of an orbit $O_{p} \subset \operatorname{Int} D$, which is either a fixed point or an orbit of higher period. On the other hand, we know 
(Theorem 2) the forms of expansions of periodic orbits. Namely, $\operatorname{reg}_{n}(q)$ could be realized by indices of:

1) $n$-orbit of the form $\left(\alpha^{\prime}\right)$ of Theorem 2 or

2) either by the sum of some indices of $n$-orbits of the form $\operatorname{reg}_{n}(q)$ in $\left(\beta^{\prime}\right)$; or an orbit of a $\gamma_{n}$-periodic point in $\left(\beta^{\prime}\right)$.

Remark 2 In fact from the above proof we can deduce that in case there are no $\gamma_{n}$-fixed points in $\operatorname{Int} D$, then in addition to a periodic point there must be also a fixed point in $\operatorname{Int} D$, as its periodic expansion must realize $\operatorname{reg}_{1}(q)$ appearing in the left-hand side of (18).

\section{Cylindrical Flux Tube with Cylindrical Obstacle Inside: An Annulus Case}

\subsection{Topological Constraints Determined by Fixed Point Indices of Iterations}

In this part of our work we consider the case of the disk with a hole (annulus), i.e. the situation where some (cylindrical) solid obstacle is placed within the tube. We assume this obstacle is homogenous in shape along the whole tube so that any plane

Fig. 1 The scheme of a cylindrical magnetic flux tube with one cylindrical obstacle, leading to a field line mapping between two annuli spaces

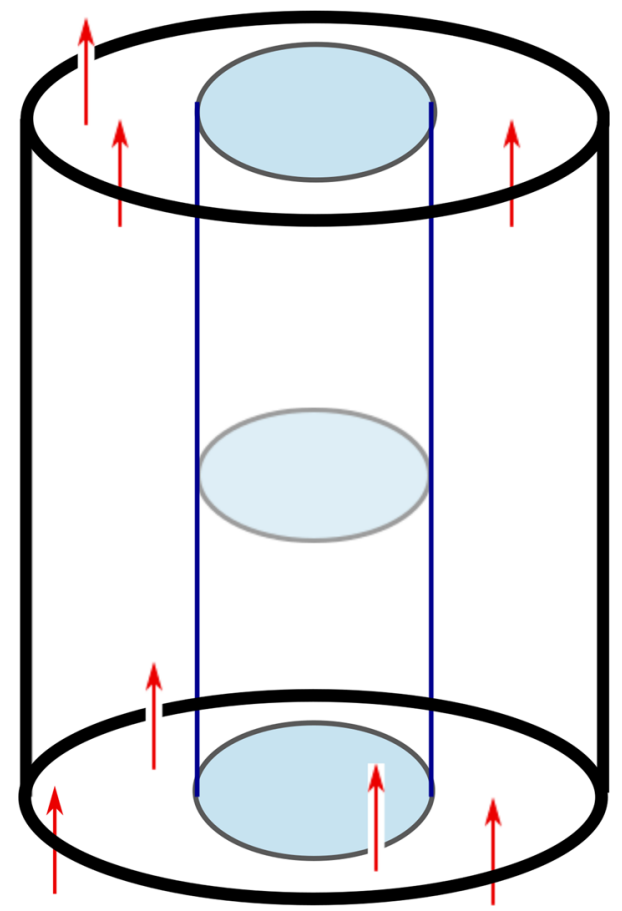


parallel to the base of the cylinder introduces the same section of the tube (see Fig. 1). The conditions (1a)-(1d') are now translated into the following:

$$
\begin{aligned}
& \mathbf{B} \neq 0, \\
& \left.B^{r}\right|_{r=R_{1}}=\left.B^{r}\right|_{r=R_{2}}=0, \\
& B^{z}(r, \varphi, z)>0 \forall(r, \varphi, z), \\
& \mathbf{B}(r, \varphi, z)=\mathbf{B}(r, \varphi, z+1) \text { for all }(r, \varphi, z) \text { such that } R_{1} \geqslant r \geqslant R_{2} \text { and } z \in[0, \infty),
\end{aligned}
$$

where $R_{1}$ and $R_{2}\left(R_{1}<R_{2}\right)$ denote the radius of the base of the interior and exterior cylinder, respectively, and $A_{0}$ and $A_{1}$ are the lower and upper annulus (for $z=0$ and $z=1$, respectively) in the base and "top"-section of the tube. Since $A_{0}=A_{1}$ when dropping the $z$-coordinate, we will write simply $A_{0}=A_{1}=A$, with $\partial A=S_{1} \cup S_{2}$, $S_{1}$ and $S_{2}$ denoting the two circles with radius $R_{1}$ and $R_{2}$, correspondingly. Similarly as before, we obtain a map $f: A \rightarrow A$ (given by the formula (5)) which is an orientation preserving diffeomorphism of $A$. Therefore $f$ preserves the boundary $\partial A$. We recall that in general there exist orientation preserving diffeomorphims of $A$, which exchange boundary components (e.g. $f(r, \varphi)=\left(\sqrt{R_{1}^{2}+R_{2}^{2}-r^{2}},-\varphi\right)$ ). However, the condition (19b) implies that when $f$ is a field line mapping obtained from our vector field, then $f$ preserves boundary components.

The following lemma holds:

Lemma 4 Let $f: A \rightarrow A$ be an orientation preserving diffeomorphism. We assume that for every $q \in \mathbb{N}$ the set Fix $\left(f^{q}\right)$ is finite. Then

$$
T_{i n t}^{q}+T_{\partial}^{q}=0
$$

for all $q \in \mathbb{N}$.

Proof We use the well-known version of Poincaré-Hopf theorem, stating that for a homeomorphism $f$ of a manifold $M$ the following relation holds (cf. [16]):

$$
\chi(M)=\sum_{x_{0} \in \operatorname{Fix}(f)} \operatorname{ind}\left(f, x_{0}\right),
$$

where $\chi$ denotes the Euler characteristics of $M$.

In our case, $\chi(A)=\chi\left(S^{1}\right)=0$. Of course, the same property holds for every iteration $f^{q}$, which yields the formula (20).

Denote by $x_{i}$ periodic points of $f$ with minimal period $k_{1}$ in $S_{1} \subset \partial A$ and by $y_{j}$ periodic points of $f$ with minimal period $k_{2}$ in $S_{2} \subset \partial A$. Then the sequence of indices of $\left(f^{k_{1}}\right)^{q}$ at each $x_{i}$ or $\left(f^{k_{2}}\right)^{q}$ at $y_{j}$ is constant by Lemma 1 . Thus, $T_{\partial}^{q}=$ $\sum_{i} a_{k_{1}}^{i} \operatorname{reg}_{k_{1}}(q)+\sum_{j} a_{k_{2}}^{j} \operatorname{reg}_{k_{2}}(q)$. We obtain the counterpart of Theorem 3 (with an analogous proof, which we omitt).

Theorem 5 If $f$ is the field line map of the magnetic field over a cylindrical flux tube with a cylindrical obstacle inside, then 
a) if $T_{\mathrm{int}}^{1} \neq 0$, then $T_{\mathrm{int}}^{q}=T_{\mathrm{int}}^{1} \neq 0$ for all $q \in \mathbb{N}$,

b) if $T_{\mathrm{int}}^{1}=0$, then $T_{\mathrm{int}}^{q}=0$ for all $q \in \mathbb{N}$ iff $\sum_{i} a_{k_{1}}^{i}=\sum_{j} a_{k_{2}}^{j}=0$.

Again in the case b) of Theorem 5 if the condition $\sum_{i} a_{k_{1}}^{i}=\sum_{j} a_{k_{2}}^{j}=0$ is not satisfied we obtain additional constraints for the magnetic flow. Assume that $\sum_{i} a_{k_{1}}^{i} \neq 0$ and $\sum_{j} a_{k_{2}}^{j} \neq 0$ (if only one of the sum is non-zero an analogous statement with one period applies). Then there are some periodic points of minimal period $k_{1}$ in $S_{1}$ and $k_{2}$ in $S_{2}$. Repeating the reasoning contained in the proof of Theorem 4 with two terms $A_{k_{1}} \operatorname{reg}_{k_{1}}(q)$ and $A_{k_{2}} \operatorname{reg}_{k_{2}}(q)$ in the left-hand side of (18) we get:

Theorem 6 If $f$ is the field line map of the magnetic field over cylindrical flux tube with cylindrical obstacle inside, and $k_{1}, k_{2}>1$ are the minimal periods for boundary periodic points of $f$, then $f$ has $k_{1}$-periodic point or $\gamma_{k_{1}}$-periodic point as well as $k_{2}$-periodic point or $\gamma_{k_{2}}$-periodic point in Int $D$.

Now, let us assume that all periodic points are non-degenerate and that (2) is satisfied i.e. the divergence is zero. Then, it has been proved in [23] (Lemma 3.3) that for a boundary fixed point $x_{0}$

$$
\operatorname{ind}\left(f^{q}, x_{0}\right) \in\{-1,0\} \text { and }\left(\operatorname{ind}\left(f^{q}, x_{0}\right)\right)_{q} \text { is a constant sequence. }
$$

Under these assumptions we can obtain the refinement of Theorem 6.

Theorem 7 Under the above assumptions, if $f$ is the field line mapping of the magnetic field over a cylindrical flux tube with a cylindrical obstacle inside, and $k_{1}, k_{2}>1$ are the minimal periods for boundary periodic points of $f$ with non-zero indices, then $f$ has $k_{1}$-periodic point and $k_{2}$-periodic point in IntD with non-negative indices of iterations.

Proof Let $x_{0} \in P_{m}(f) \cap \partial A$ be a periodic point with ind $\left(f^{m}, x_{0}\right) \neq 0$ (and $m=k_{1}$ or $m=k_{2}$ ). Then by the (21) we get that

$$
\operatorname{ind}\left(f^{q}, O_{x_{0}}\right)=-\operatorname{reg}_{m}(q) .
$$

Now let us establish the formula for indices of non-degenerate points located inside $A$.

Let us denote for short the derivative of $f$ at $y_{0} \in \operatorname{Fix}(f) \cap \operatorname{Int} A$ by $D=D f\left(y_{0}\right)$ and by $\sigma(D)$ its spectrum. By $\sigma_{+}$we denote the number of real eigenvalues of $D$ greater than 1 and by $\sigma_{-}$the number of real eigenvalues of $D$ less than -1 , in both cases counting with multiplicity. We get (cf. [4]):

$$
\operatorname{ind}\left(f^{q}, y_{0}\right)=\operatorname{sgn} \operatorname{det}\left(I d-D^{q}\right)=\left\{\begin{array}{cc}
(-1)^{\sigma_{+}} & \text {if } \quad q \text { is odd }, \\
(-1)^{\sigma_{+}+\sigma_{-}} & \text {if } \quad \text { q is even } .
\end{array}\right.
$$

On the other hand, as $f$ is orientation preserving, $\sigma_{-}=0$ or $\sigma_{-}=2$ is an even number. As a result, $\operatorname{ind}\left(f^{q}, y_{0}\right)= \pm \operatorname{reg}_{1}(q)$. Consequently, for $y_{0} \in P_{m}(f) \cap \operatorname{Int} A$

$$
\operatorname{ind}\left(f^{q}, O_{y_{0}}\right)= \pm \operatorname{reg}_{m}(q) \text {. }
$$


By the assumption there are points of minimal period $k_{1}$ and $k_{2}$ in $\partial A$. Thus, by the equation (20) and (22) we obtain:

$$
\left|A_{k_{1}}\right| \operatorname{reg}_{k_{1}}(q)+\left|A_{k_{2}}\right| \operatorname{reg}_{k_{2}}(q)=\sum_{p \in \mathrm{P}(f) \cap \operatorname{Int} D} \operatorname{ind}\left(f^{q}, p\right),
$$

where $A_{k_{1}}<0, A_{k_{2}}<0$ and $\left|A_{k_{1}}\right|,\left|A_{k_{2}}\right|$ denote the number of periodic orbits of the period $k_{1}$ or $k_{2}$ with non-zero indices on the respective boundary component of $\partial A$. Let $O^{(m)}$ denote an $m$-orbit of $f$. Applying the formula (24) we obtain:

$$
\left|A_{k_{1}}\right| \operatorname{reg}_{k_{1}}(q)+\left|A_{k_{2}}\right| \operatorname{reg}_{k_{2}}(q)=\sum_{m} \sum_{O^{(m)} \subset \operatorname{Int} D} \pm \operatorname{reg}_{m}(q),
$$

which gives the thesis.

\subsection{Topological Constraints Resulting from the Geometry of the Tube}

In this part we recall some results concerning periodic orbits and rotations numbers for homeomorphisms of annulus, as summarised in [6], and apply them in our case. As in this section we do not make use of the index theory, we omit the assumption that $\operatorname{Fix}\left(f^{q}\right)$ is finite for every $q$, i.e. we allow for non-isolated fixed and periodic points. Let us define an annulus $A$ as $A:=S^{1} \times I$, where $I=[0,1]$.

Definition 4 Let $f: A \rightarrow A$ be a homeomorphism. We say that $F: \tilde{A} \rightarrow \tilde{A}$, where $\tilde{A}:=\mathbb{R} \times I$, is a lift of $f$, if

$$
f(\bar{\pi}(x, y))=\bar{\pi}(F(x, y)),
$$

where $\bar{\pi}: \tilde{A} \rightarrow A$ is given by $\bar{\pi}(x, y)=(\mathfrak{p}(x), y)$.

In this case, $G(x, y):=F(x, y)+(m, 0), m \in \mathbb{Z}$, is also a lift of $f$.

Before we define the rotation number for an annulus map, we need to define the translation number with respect to its lift:

Definition 5 Let $f: A \rightarrow A$ be an orientation preserving homeomorphism which preserves boundary components and let $F: \tilde{A} \rightarrow \tilde{A}$ be its lift. The translation number of a point $w=(x, y) \in \tilde{A}$ under $F$ is defined as

$$
\tau(w, F):=\lim _{n \rightarrow \infty} \frac{p_{1}\left(F^{n}(w)-w\right)}{n},
$$

(provided that the limit exists), where $p_{1}: \tilde{A} \rightarrow \mathbb{R}$ is the projection on the first coordinate. Now, the rotation number of $\bar{\pi}(w)$ under $f$ is the element of $S^{1}=\mathbb{R} / \mathbb{Z}$ defined by

$$
\varrho(\bar{\pi}(w), f):=\mathfrak{p}(\tau(w, F)) .
$$

We remark that if $\tau(w, F)$ exists then $\varrho(\bar{\pi}(w), f)$ exists as well and is well defined, i.e. does not depend on the choice of a lift $F$.

Note also that since we identify $S^{1}$ with $\mathbb{R} / \mathbb{Z}$, the projection $\mathfrak{p}$ is equivalent to taking $\bmod 1$, i.e. one can write $\varrho(\bar{\pi}(w), f)=\tau(w, F) \bmod 1$. 
For an annulus homeomorphism the rotation number $\varrho(\bar{\pi}(w), f)$ might not exist, and even if it exists it might depend on the point $\bar{\pi}(w) \in A$ at which it is calculated. Nevertheless, it still has some good properties [6]:

- periodic orbits have rational rotation numbers

- if $f$ preserves a probability Borel measure $\mu$, then $\varrho(x, f)$ exists for all $x \in A$, except a set of $\mu$ measure zero. Moreover,

$$
\tau_{\mu}(F):=\int_{S^{1}} \tau(x, F) d \mu=\int_{S^{1}} \Phi d \mu,
$$

where $\Phi: A \rightarrow \mathbb{R}, \Phi(\bar{\pi}(w))=p_{1}(F(w)-w)$ is the displacement function of $F$. The value of $\tau_{\mu}(F)$ is called the mean translation number of $F$.

Definition 6 If $f: A \rightarrow A$ is an orientation preserving homeomorphism, which preserves boundary components and $F: \tilde{A} \rightarrow \tilde{A}$ is its lift, then the collection of all translation numbers of points (for which the translation number exists)

$$
\operatorname{rot}(F):=\{\tau(w, F): w \in \tilde{A}\}
$$

is called the rotation set of $f$.

The set $\operatorname{rot}(F)$ is closed. Moreover, if we further assume that our map $f$ is also area-preserving, then the rotation number tool is especially interesting. In particular, the rotation set is in this case a closed interval (see e.g. remarks following Theorem 4.3 in [6]) and the following holds:

Theorem 8 (cf. [6]) Suppose that $f: A \rightarrow A$ is an area preserving homeomorphism which preserves orientation and boundary components. Let $F: \tilde{A} \rightarrow \tilde{A}$ be its lift. If there exist two points $w_{0}, w_{1} \in \tilde{A}$ such that

$$
\tau\left(w_{0}, F\right) \neq \tau\left(w_{1}, F\right)
$$

then $f$ has infinitely many periodic points. Moreover, the set of minimal periods of these periodic points is infinite.

The above theorem is a compilation of Theorem 4.1, Theorem 4.2, Theorem 5.4 and Theorem 5.7 from [6]. It can be justified by the properties of the rotation set: precisely, if $\tau\left(w_{0}, F\right) \neq \tau\left(w_{1}, F\right)$, then the rotation interval $\operatorname{rot}(F)$ is non-degenerate since it contains the non-trivial interval $\left[\tau\left(w_{0}, F\right), \tau\left(w_{1}, F\right)\right]$ and every number $\tau \in$ $\operatorname{rot}(F)$ is realized as the translation number $\tau(w, F)$ for some $w \in \tilde{A}$. As the rational translation numbers correspond to periodic orbits, the statement about period points follows. In particular, if $p / q \in \mathbb{Q}$ (where $p / q$ is in lowest terms) satisfies

$$
\tau\left(w_{0}, F\right) \leqslant p / q \leqslant \tau\left(w_{1}, F\right),
$$

then there exist at least two distinct periodic orbits with minimal periods $q$, as follows from Theorem 4.2 in [6].

We also remark that if $F_{0}(x):=F(x, 0)$ and $F_{1}(x):=F(x, 1)$ denote the lifts of the induced circle homeomorphisms $f_{0}=F_{0} \bmod 1$ and $f_{1}=F_{1} \bmod 1$ at 
the boundary components of $A$, then the unique rotation numbers $\varrho\left(f_{0}\right)$ and $\varrho\left(f_{1}\right)$ of these circle homeomorphisms coincide with rotation numbers of boundary points under annulus homeomorphism $f$, i.e. $\varrho\left(f_{0}\right)=\tau\left(w_{0}, F\right) \bmod 1$ and $\varrho\left(f_{1}\right)=$ $\tau\left(w_{1}, F\right)$ mod 1 , where $\bar{\pi}\left(w_{0}\right)$ and $\bar{\pi}\left(w_{1}\right)$ are arbitrary points on the corresponding boundary components of $A\left(w_{0}=\left(x_{0}, 0\right) \in \tilde{A}\right.$ and $\left.w_{1}=\left(x_{1}, 1\right) \in \tilde{A}\right)$. Denoting these unique translation numbers as $\tau\left(F_{0}\right):=\tau\left(w_{0}, F\right)$ and $\tau\left(F_{1}\right):=\tau\left(w_{1}, F\right)$, it is often convenient to check whether $\tau\left(F_{0}\right) \neq \tau\left(F_{1}\right)$ in order to apply Theorem 8 .

Another classical result that may be applied in our developments is the following:

Theorem 9 (cf. Theorem 3.5 in [7]) Let $f: A \rightarrow A$ be an area preserving homeomorphism, isotopic to the identity. If $f$ has at least one fixed or periodic point, then $f$ must have infinitely many interior periodic points.

One can formulate a variety of conditions for the field $\mathbf{B}$, each of which implies that the arising map $f: A \rightarrow A$ is area preserving. Under the additional assumption (2), this holds, for example, when

$$
B^{z}\left(f\left(x_{0}\right)\right)=B^{z}\left(x_{0}\right)
$$

for every field line $f$ and every $x_{0} \in A$. Indeed, then

$$
|\mathrm{D} f|\left(x_{0}\right)=\frac{B^{z}\left(x_{0}\right)}{B^{z}\left(f\left(x_{0}\right)\right)}=1
$$

at every point $x_{0}$ (cf. [23]). Of course, in this case $f$ preserves also Lebesgue measure $\Lambda$, and therefore the rotation number exists and is unique for almost all $x \in A$. Below we provide some explicit condition for the map $f: A \rightarrow A$ to be area-preserving.

Proposition 1 Let $f: A \rightarrow A$ be the field line mapping of the magnetic field $\hat{\mathbf{B}}$ defined by the (4) associated with the field $\mathbf{B}$ satisfying our assumptions (19a)-(19d). Moreover, we assume that $\operatorname{div} \mathbf{B}(r, \varphi, z)=0$ and that

$$
\forall_{(r, \varphi, z)} r B^{r}(r, \varphi, z) \frac{\partial B^{z}}{\partial r}(r, \varphi, z)+B^{\varphi}(r, \varphi, z) \frac{\partial B^{z}}{\partial \varphi}(r, \varphi, z)=0 .
$$

Then the induced map $f: A \rightarrow A$ preserves Lebesgue measure $\Lambda$, i.e. $f$ is area preserving.

In particular, the rotation number $\varrho(x, f)$ exists for all $x \in A$, the mean rotation number $\tau_{\mu}(F)$ (where $\mu$ is the measure $\Lambda$ normalised over $A$ ) exists as well and Theorems 8 and 9 apply.

Proof Consider the three-dimensional flow $\Phi^{t}$ induced by (4). This flow, on the account of the Liouville's Theorem (see e.g. Lemma 2.4 in [22]), preserves (threedimensional) Lebesgue measure, i.e.

$$
\Lambda_{3}(C)=\Lambda_{3}\left(\Phi^{t}(C)\right)
$$


for any $t$ and Lebesgue-measurable subset $C$ of the tube, since the divergence $\operatorname{div} \hat{\mathbf{B}}=$ 0 . Indeed calculating div $\hat{\mathbf{B}}$ we obtain

$$
\begin{aligned}
\operatorname{div} \hat{\mathbf{B}}= & \frac{1}{r B^{z}(r, \varphi, z)}\left[r \frac{\partial B^{r}}{\partial r}(r, \varphi, z)+B^{r}(r, \varphi, z)+\frac{\partial B^{\varphi}}{\partial \varphi}(r, \varphi, z)\right]+ \\
& -\frac{1}{r\left(B^{z}(r, \varphi, z)\right)^{2}}\left[r B^{r}(r, \varphi, z) \frac{\partial B^{z}}{\partial r}(r, \varphi, z)+B^{\varphi}(r, \varphi, z) \frac{\partial B^{z}}{\partial \varphi}(r, \varphi, z)\right] .
\end{aligned}
$$

The first term in square brackets above vanishes since $\operatorname{div} \mathbf{B}=0$ and the second one is exactly the condition (28).

Now we show that also $f: A_{0} \rightarrow A_{1}$ preserves (two-dimensional) Lebesgue measure, i.e. $\Lambda_{2}\left(N_{0}\right)=\Lambda_{2}\left(f\left(N_{0}\right)\right)$, where $N_{0}$ is an arbitrary open set contained in $A_{0}$.

Let us denote by $N_{t}$ an open set which is the image of $N_{0}$ under $\Phi^{t}\left(N_{t} \subset A_{t}\right.$, with $\left.A_{t}=\Phi^{t}\left(A_{0}\right)\right)$. For a given $\varepsilon$ we define

$$
N_{t}^{\varepsilon}=\left\{(x, z): x \in N_{t}, t \leqslant z \leqslant t+\varepsilon\right\} .
$$

Observe that by the formula (29) for a fixed $\varepsilon$ we have: $\Lambda_{3}\left(N_{t}^{\varepsilon}\right)=$ const. for all $t$. In particular,

which implies

$$
\Lambda_{3}\left(N_{0}^{\varepsilon}\right)=\Lambda_{3}\left(N_{1}^{\varepsilon}\right)
$$

$$
\lim _{\varepsilon \rightarrow 0} \frac{\Lambda_{3}\left(N_{0}^{\varepsilon}\right)}{\varepsilon}=\lim _{\varepsilon \rightarrow 0} \frac{\Lambda_{3}\left(N_{1}^{\varepsilon}\right)}{\varepsilon}
$$

i.e. $\Lambda_{2}\left(N_{0}\right)=\Lambda_{2}\left(N_{1}\right)$.

We also remark that the condition (28) is not very restrictive in fact. In particular, all the examples studied in [23] satisfy it trivially, since always $B^{z} \equiv 1$ (and $B^{r}$ and $B^{\varphi}$ do not depend on $z$ ) therein.

\subsection{Examples}

At the end we present two examples, which allow for easy computation of translation numbers of points in $\partial A$ and hence for drawing conclusions on the existence of periodic points in $\operatorname{Int} A$.

Example 1 As the first example, let us consider vector field $\mathbf{B}=\left(B^{r}, B^{\varphi}, B^{z}\right)$ with

$$
\left\{\begin{array}{l}
B^{r}(r, \varphi, z)=(r-1)^{2}(2-r)^{2} \\
B^{\varphi}(r, \varphi, z)=\frac{2 \pi}{3}(2-r)+\frac{4 \pi}{3}(r-1)-\varphi(r-1)(2-r)[(r-1)(2-r)+2 r(3-2 r)] \\
B^{z}(r, \varphi, z)=1
\end{array}\right.
$$

The field $\mathbf{B}$ readily satisfies all the assumptions of Proposition 1 and induces a field line mapping $f: A \rightarrow A$ of the annulus with radii $R_{1}=1$ and $R_{2}=2$, which is a diffeomorphism, preserving orientation, boundary components and Lebesgue measure. Moreover, on these circles $\left\{r=R_{1}\right\}$ and $\left\{r=R_{2}\right\}, f$ reduces, correspondingly, to the rotation by $\frac{2 \pi}{3}$ (i.e. $1 / 3$ of the full angle $2 \pi$ ) and to the rotation by $\frac{4 \pi}{3}$. Indeed, $B^{\varphi}(1, \varphi)=\frac{2 \pi}{3}$ and $B^{\varphi}(2, \varphi)=\frac{4 \pi}{3}$ and $\Phi(\varphi, z)=\varphi+\frac{2 \pi}{3} z$ and $\Phi(\varphi, z)=\varphi+\frac{4 \pi}{3} z$ are the flows induced on $\left\{\left(R_{1}, \varphi, z\right)\right\}$ and $\left\{\left(R_{2}, \varphi, z\right)\right\}$, respectively. It follows that the 
rotation number $\tau(\omega)=1 / 3$ for every point $\omega$ on the internal circle $\left\{r=R_{1}\right\}$ and $\tau(\omega)=2 / 3$ on the external circle $\left\{r=R_{2}\right\}$ (under the projection $\varphi \mapsto \exp (\iota \varphi)$ ). Thus on the account of Theorem 8 , the set of minimal periods of $f$ is infinite. Moreover, as one concludes from Theorem 4.3 in [6] (see also [15]), every number $\tau \in[1 / 3,2 / 3]$ is a translation number for some point $w \in \tilde{A}$ (the closed rotation interval $\operatorname{rot}(f):=\{\tau(w, F): w \in \tilde{A}\}$ contains the interval $[1 / 3,2 / 3])$.

Therefore, for any co-prime $p, q \in \mathbb{N}, 1 / 3 \leqslant p / q \leqslant 2 / 3, f: A \rightarrow A$ has a periodic point with minimal period $q$.

Example 2 Let $\mathbf{B}$ be the so-called hyperbolic vector field (see also [23]) with $B^{z} \equiv 1$ and

$$
\mathbf{B}^{\text {hyp }}(r, \varphi)=\left(2 \cos (2 \varphi) \sin \left(\frac{\pi r}{2}\right),-\sin (2 \varphi)\left[\sin \left(\frac{\pi r}{2}+\frac{\pi r}{2} \cos \frac{\pi r}{2}\right)\right]\right) .
$$

It is immediate to check that the conditions listed in Proposition 1 hold, with $R_{1}=2$ and $R_{2}=4$. On the invariant circle $\left\{r=R_{1}\right\}$ we obtain:

$$
B^{\varphi}(2, \varphi)=\pi \sin (2 \varphi)
$$

whereas on $\left\{r=R_{2}\right\}$ we have:

$$
B^{\varphi}(4, \varphi)=-2 \pi \sin (2 \varphi)
$$

Thus we readily see that $0 \equiv 2 \pi, \frac{\pi}{2}, \pi, \frac{3 \pi}{2}$ are the fixed points on both these circles and both translation numbers (as verified also numerically) vanish: $\tau\left(F_{0}\right)=$ $\tau\left(F_{1}\right)=0$. (It is also possible to obtain the exact formulas for $F_{0}$ and $F_{1}$ by solving corresponding one-dimensional differential equations on the intervals between the nearby fixed points).

However from the existence of these (boundary) fixed points, it follows (on the account of Theorem 9) that that there are infinitely many interior periodic points as well.

Open Access This article is distributed under the terms of the Creative Commons Attribution 4.0 International License (http://creativecommons.org/licenses/by/4.0/), which permits unrestricted use, distribution, and reproduction in any medium, provided you give appropriate credit to the original author(s) and the source, provide a link to the Creative Commons license, and indicate if changes were made.

\section{References}

1. Arango, J., Gómez, A.: Flows and diffeomorphisms. Rev. Colombiana Mat. 32, 13-27 (1998)

2. Babenko, I.K., Bogatyi, S.A.: The behavior of the index of periodic points under iterations of a mapping. Math. USSR Izv. 38, 1-26 (1992)

3. Berger, M.A., Field, G.B.: The topological properties of magnetic helicity. J. Fluid. Mech. 147, 133148 (1984)

4. Chow, S.N., Mallet-Parret, J., Yorke, J.A.: A periodic point index which is a bifurcation invariant, Geometric dynamics (Rio de Janeiro, 1981), pp. 109-131. Springer Lecture Notes in Math, Berlin (1983). 1007

5. Fetter, A.L.: Rotating trapped Bose-Einstein condensates. Rev. Mod. Phys. 81, 647-691 (2009)

6. Franks, J.: Rotation numbers and instability sets. Bull. Amer. Math. Soc. (N.S.) 40, 263-279 (2003)

7. Franks, J.: Geodesics on $S^{2}$ and periodic points of annulus homeomorphisms. Invent. Math. 108, 403-418 (1992) 
8. Freund, I.: Critical point explosions in two-dimensional wave fields. Opt. Commun. 159, 99-117 (1999)

9. Graff, G.: Minimal number of periodic points for smooth self-maps of two-holed 3-dimensional closed ball. Topol. Method Nonl. Ann. 33(1), 121-130 (2009)

10. Graff, G.: Minimal periods of maps of rational exterior spaces. Fund. Math. 163(2), 99-115 (2009)

11. Graff, G., Jezierski, J.: Minimal number of periodic points of smooth boundary-preserving self-maps of simply-connected manifolds. Geom. Dedicata 187(1), 241-258 (2017)

12. Graff, G., Jezierski, J.: Minimal number of periodic points for $C^{1}$ self-maps of compact simplyconnected manifolds. Forum Math. 21(3), 491-509 (2009)

13. Graff, G., Nowak-Przygodzki, P.: General form of fixed point indices of an iterated $C^{1}$ map and infiniteness of minimal periods. Dyn. Syst. 23(4), 491-504 (2008)

14. Katok, A., Hasselblatt, B.: Introduction to the Modern Theory of Dynamical Systems Encyclopedia of Mathematics and its Applications, vol. 54. Cambridge University Press, Cambridge (1995)

15. Handel, M.: The rotation set of a homeomorphism of the annulus is closed. Comm. Math. Phys. 127, 339-349 (1990)

16. Jezierski, J., Marzantowicz, W.: Homotopy Methods in Topological Fixed and Periodic Points Theory Topological Fixed Point Theory and its Applications, vol. 3. Springer, Dordrecht (2006)

17. Matsuoka, T., Shiraki, H.: Smooth maps with finitely many periodic points. Mem. Fac. Sci., Kochi Univ. (Math) 11, 1-6 (1990)

18. Pontin, D.I., Wilmot-Smith, A.L., Hornig, G., Galsgaard, K.: Dynamics of braided coronal loops. II. Cascade to multiple small-scale reconnection events. Astron. Astrophys. 525, A57 (2011)

19. Rubin, J.E., Signerska-Rynkowska, J., Touboul, J.D., Vidal, A.: Wild oscillations in a nonlinear neuron model with resets: (II) Mixed-mode oscillations. Discrete Contin. Dyn. Syst. Ser. B 22(10), 40034039 (2017)

20. Taylor, J.B.: Relaxation of toroidal plasma and generation of reverse magnetic fields. Phys. Rev. Lett. 33, 1139 (1974)

21. Class for Physics of the Royal Swedish Academy of Sciences, Topological phase transitions and topological phases of matter. Scientifc Background on the Nobel Prize in Physics (2016)

22. Verhulst, F.: Nonlinear Differential Equations and Dynamical Systems. Springer, Berlin (1996)

23. Yeates, A.R., Hornig, G.: Dynamical constraints from field line topology in magnetic flux tubes. J. Phys. A: Math. Theor. 44, 265501 (2011)

24. Yeates, A.R., Hornig, G., Wilmot-Smith, A.L.: Topological constraints on magnetic relaxation. Phys. Rev. Lett. 105, 085002 (2010) 